Artigo Original

Original Article

Debora Maria Befi-Lopes ${ }^{1}$

Letícia Bondezan Bacchin ${ }^{1}$

Paula Renata Pedott ${ }^{1}$

Ana Manhani Cáceres-Assenço

Descritores

Linguagem infantil

Transtornos do desenvolvimento da linguagem Narração

Fonoaudiologia

Desenvolvimento da linguagem

Keywords

Child language

Language development disorders

Narration

Speech, language and hearing sciences Language development

Endereço para correspondência: Debora Maria Befi-Lopes

R. Cipotânea, 51, Cidade Universitária, São Paulo (SP), Brasil, CEP: 05360-160. E-mail:dmblopes@usp.br

Recebido em: 13/05/2013

Aceito em: 02/08/2013

\section{Complexidade da história e pausas silentes em crianças com e sem distúrbio específico de linguagem}

\author{
Story's complexity and silent pauses in children with and \\ without specific language impairment
}

Objetivo: Verificar o tempo médio das pausas silentes em narrativas e a influência da complexidade da história na produção destas pausas nas narrativas de crianças em desenvolvimento típico de linguagem e em crianças com DEL; além de comparar estes aspectos nos dois grupos. Métodos: Participaram do estudo 60 crianças, entre sete e dez anos, sendo 40 em desenvolvimento típico de linguagem e 20 com DEL. Para a coleta de dados foi utilizada uma série de 15 histórias, representadas por figuras, compostas por quatro cenas cada. As histórias apresentam complexidade crescente a partir das relações entre os personagens, desde ausência de intencionalidade (sequências mecânicas e comportamentais) até relações entre os personagens com atribuição de estados mentais (sequências intencionais), possibilitando o registro do tempo médio das pausas silentes produzidas nas narrativas. Resultados: A complexidade da história influiu no tempo médio de pausas silentes nas narrativas das crianças em desenvolvimento típico de linguagem, porém, para as crianças com DEL não foi observado tal padrão. A comparação entre os grupos indica diferença significativa em todos os tipos de narrativas, com maior média no grupo com DEL. Conclusão: As crianças com DEL, devido ao seu comprometimento linguístico, apresentaram pausas silentes mais longas em suas narrativas. A complexidade da história influenciou o tempo médio da pausa silente durante a produção das narrativas das crianças em desenvolvimento típico de linguagem, porém esta influência não ocorreu nas narrativas das crianças com DEL.

\begin{abstract}
Purpose: To verify the average time of silent pauses in narratives and the influence of story's complexity in the occurrence of these pauses in narratives of children with typical language development and children with specific language impairment (SLI), and further to compare these aspects between groups. Methods: Sixty children aged between seven to ten years took part in this research, being 40 typical language developing children and 20 with SLI. To collect data, each child produced 15 narratives, each one based on a four-scene-sequence. These narratives show increasing complexity of the relations between the characters, since absence of intentionality (mechanical and behavioral sequences) to relations between characters with mental states attribution (intentional sequences), which allowed the survey of the average time of silent pauses in the narratives produced. Results: Story's complexity has influenced the average time of silent pauses in narratives of children with typical language development, however, for children with SLI this pattern was not observed. The comparison between groups indicates a significant difference in all types of narratives, with the highest average in the group with SLI. Conclusion: Due to their linguistic impairment, children with SLI had longer silent pauses in their narratives. Story's complexity has influenced the average time of silent pauses in narratives of children with typical language development, but this difference hasn't occurred in SLI children's narratives.
\end{abstract}

Trabalho realizado no Laboratório de Investigação Fonoaudiológica em Desenvolvimento da Linguagem e suas Alterações do Curso de Fonoaudiologia da Faculdade de Medicina da Universidade de São Paulo - USP São Paulo (SP), Brasil.

(1) Curso de Fonoaudiologia da Faculdade de Medicina da Universidade de São Paulo - USP - São Paulo (SP), Brasil Fonte de financiamento: Este artigo apresenta resultados de três projetos financiados pela Fundação de Amparo à Pesquisa do Estado de São Paulo (FAPESP) por meio dos processos 2009/14045-0 e 2010/07613-0 e 2010/07637-6

Conflito de interesses: nada a declarar 


\section{INTRODUÇÃO}

A produção da narrativa envolve coordenação entre as habilidades morfossintáticas e semânticas dentro de um sistema que inclui aspectos pragmáticos e convenções culturais, que influenciam a maneira como a informação deve ser apresentada ao ouvinte ${ }^{(1)}$.

Para que a produção de fala seja fluente é preciso que informações semânticas, fonológicas e sintáticas sejam ativadas em harmonia com a informação contextual ${ }^{(2)}$, e que várias partes do trato vocal sejam movimentadas de forma suave e precisa ${ }^{(3)}$. Portanto, quando falhas ocorrem neste processo, a fluência na produção é interrompida, produzindo rupturas na fala ${ }^{(4)}$.

Em desenvolvimento típico de linguagem, a ocorrência de rupturas de fala pode estar relacionada ao descompasso entre habilidades sintáticas e lexicais durante períodos específicos do desenvolvimento ${ }^{(5)}$, relacionando-se com o surgimento de estruturas mais complexas durante a aquisição da gramática, principalmente com aquelas ainda não totalmente compreendidas pela criança ${ }^{(6)}$. Logo, elas seriam utilizadas como estratégias de processamento para ganhar tempo ou reparar erros durante a produção de sentenças ${ }^{(4,7,8)}$.

Durante o desenvolvimento lexical, a representação inicial de aspectos semânticos e fonológicos de palavras desconhecidas é incompleta e limitada, necessitando de experiências posteriores para se fortalecer ${ }^{(9,10)}$. Contudo, quanto mais experiência a criança tiver com uma palavra até então desconhecida, mais forte ficarão suas representações fonológicas e semânticas e ela precisará de menos esforço para acessar a palavra na memória, produzindo, consequentemente, menos rupturas de fala ${ }^{(5,10,11)}$.

Dentre estas rupturas, a pausa silente, ruptura gaga caracterizada por silêncio com duração maior ou igual a $250 \mathrm{~ms}$, é apontada na literatura como uma estratégia de ganho de tempo para formulação do enunciado sem adição de palavras. Ela costuma ocorrer quando há sobrecarga de informações relacionadas ao processamento linguístico ou quando o falante encontra dificuldades na formulação de conceitos e na ativação e recuperação de padrões sintáticos, semânticos e lexicais ${ }^{(4)}$.

Crianças com distúrbio específico de linguagem (DEL) produzem enunciados mais curtos que seus pares em desenvolvimento típico, indicando que utilizam estruturas mais simples para se comunicar. Estas crianças têm comprometimento linguístico que não pode ser atribuído à deficiência auditiva, à disfunção neuromotora, à deficiência mental e aos transtornos invasivos de desenvolvimento ${ }^{(12,13)}$.

A análise de elementos de narrativas de crianças com DEL confirma que elas são deficientes em aspectos linguísticos anteriores à elaboração expressiva, demoram mais para aprender a língua materna e enfrentam dificuldades na alfabetização $0^{(14)}$.

Em geral, tais crianças apresentam grande sobrecarga no sistema de processamento da informação linguística quando são submetidas a uma tarefa de produção mais complexa e extensa. Esta sobrecarga é explicada pelos déficits lexicais iniciais importantes que permanecem ao longo do desenvolvimento, principalmente nas palavras de classe fechada, pelas falhas na formação das regras gramaticais da língua e pela desorganização do sistema fonético-fonológico para formação de regras morfológicas ${ }^{(15)}$.

Assim, crianças com DEL apresentam déficits na elaboração do discurso devido ao restrito conhecimento da estrutura da língua, a dificuldades na organização textual, compreensão da temporalidade e relações de causa e efeito, além de restrito conhecimento estrutural. Apresentam, portanto, narrativas mais rudimentares se comparadas a seus pares cronológicos em desenvolvimento típico de linguagem, independente do tipo de história fornecida ${ }^{(16)}$.

A partir do exposto, este estudo teve como objetivo verificar o tempo médio das pausas silentes em narrativas e a influência da complexidade da história na produção destas pausas nas narrativas de crianças em desenvolvimento típico de linguagem e em crianças com DEL, além de comparar estes aspectos nos dois grupos.

\section{MÉTODOS}

O projeto foi aprovado pela comissão de ética da instituição Faculdade de Medicina da Universidade de São Paulo (USP) com o número 1150/09, e os responsáveis pelas crianças assinaram o Termo de Consentimento Livre e Esclarecido.

O estudo contou com a participação de 40 crianças em desenvolvimento típico de linguagem (18 do gênero masculino) e 20 com DEL (14 do gênero masculino), com idade entre sete e 10 anos. Os sujeitos foram pareados por faixa etária, sendo que para cada um do grupo com DEL deveria haver dois do grupo em desenvolvimento típico. Todos os sujeitos residiam na cidade de São Paulo e estudavam em sala regular de escola pública.

A seleção dos sujeitos em desenvolvimento típico de linguagem foi realizada em uma escola estadual localizada na Zona Oeste da cidade de São Paulo. Inicialmente, todos eles foram triados individualmente durante seu período regular de aula e em sala reservada para esta pesquisa.

Os critérios de inclusão do grupo em desenvolvimento típico foram: não apresentar queixas relativas ao desenvolvimento da linguagem; frequentar sala comum e compatível com a faixa etária; não apresentar nenhum processo fonológico produtivo ou trocas idiossincráticas nas provas de imitação e nomeação da Fonologia ${ }^{(17)}$; e desempenho compatível com o esperado em tarefas que envolvem consciência fonológica, leitura e escrita ${ }^{(18)}$.

Os integrantes do grupo com DEL estavam em atendimento fonoaudiológico semanal e já haviam sido diagnosticados anteriormente com base em critérios internacionais - déficit exclusivamente linguístico e quociente intelectual (QI) dentro da normalidade ${ }^{(12)}$. Para tal diagnóstico, a criança deveria apresentar audição preservada e resultados abaixo do esperado em pelo menos dois testes padronizados de linguagem, da bateria de avaliação de linguagem infantil $\mathrm{ABFW}^{(19)}$ e da avaliação da extensão média do enunciado ${ }^{(15)}$. O tempo mínimo de terapia fonoaudiológica destes sujeitos era de seis meses e a média três anos.

Para a coleta de dados foi utilizada uma série de 15 histórias, representadas por figuras, compostas por quatro cenas cada. Elas foram classificadas segundo as relações envolvidas 
entre as personagens, e sua complexidade foi gradualmente aumentada $^{(20,21)}$ :

- Mecânica 1: objetos interagindo casualmente uns com os outros;

- Mecânica 2: pessoas e objetos atuando casualmente uns com os outros;

- Comportamental 1: uma pessoa atuando em situações rotineiras, que não requerem atribuição de estados mentais;

- Comportamental 2: pessoa atuando em situações sociais rotineiras, envolvendo mais de uma pessoa, que não requerem atribuição de estados mentais;

- Intencional: pessoa atuando em atividades diárias que requerem atribuição de estados mentais.

Durante a coleta de dados, era explicado aos participantes que a sequência de quatro figuras compunha uma história. A primeira era apresentada à criança e esta era solicitada a organizar as demais e narrar a respectiva história. Caso as figuras não fossem ordenadas da forma usual, a pesquisadora anotava a ordem utilizada, mas não solicitava à criança que reorganizasse ou narrasse conforme a ordem usual, pois assim todas elas elaborariam histórias compatíveis com a sua compreensão da sequência. Para eliminar possíveis variáveis, como déficit de memória de curto prazo, as figuras ficaram visíveis para a criança durante todo o tempo de sua narração. Os dados referentes à ordenação das figuras não foi considerado nesta etapa da pesquisa.

Cada história narrada foi transcrita e, posteriormente, submetida à análise das pausas silentes em software desenvolvido especificamente para este fim, o qual gerou um relatório com o valor de cada pausa silente (em ms). Como resultado foi obtido um levantamento do tempo (em ms) das pausas imediatamente anteriores a cada palavra de cada narrativa.

Para responder aos objetivos deste estudo foi realizada uma análise estatística descritiva e inferencial no software SPSS versão 18. A ANOVA de Friedman e o teste de postos com sinais de Wilcoxon foram utilizados para verificar a influência da complexidade da história no tempo médio de pausas silentes. Já o Teste de Mann-Whitney foi empregado para comparar o tempo médio das pausas silentes entre os grupos. O nível de significância inicial adotado foi de $5 \%$.

\section{RESULTADOS}

Para facilitar a compreensão dos resultados, primeiramente será apresentado o desempenho de cada um dos grupos e, posteriormente, a comparação entre eles.

\section{Desenvolvimento típico de linguagem}

A análise descritiva obtida a partir do tempo médio de pausas silentes por história revela que a menor média ocorreu nas histórias de tipo intencional e a maior nas histórias de tipo mecânica 1 (Tabela 1).

A ANOVA de Friedman indicou que o tipo de história influi significativamente no tempo médio de pausas $\left(\chi^{2}(4)=21,58\right.$, $\mathrm{p}<0,001)$.

Para acompanhar estes achados foram utilizados testes de Wilcoxon com aplicação de uma correção de Bonferroni e testados dez efeitos planejados com nível de significância de 0,005 .

$\mathrm{O}$ ranqueamento da ANOVA de Friedman ordenou de forma crescente os tipos de história de acordo com o tempo médio de pausas silentes, da seguinte forma: intencional = mecânica 2, comportamental 2, mecânica 1 e comportamental 1 (Tabela 2).

Estes resultados indicam que não há diferença estatística apenas entre as histórias mecânica 1 e comportamental 1; mecânica 2 e comportamental 2; e mecânica 2 e intencional. Logo, foi possível verificar que o tipo de história influencia o tempo médio de pausas silentes durante a produção de narrativas para as crianças em desenvolvimento típico de linguagem.

\section{Distúrbio Específico de Linguagem}

Para verificar a influência do tipo de história, a análise descritiva obtida a partir do tempo médio de pausas silentes revela que a menor média ocorreu nas histórias de tipo intencional e a maior nas histórias de tipo comportamental 1 (Tabela 3).

A ANOVA de Friedman indicou que o tipo de história não influi significativamente no tempo médio de pausas $\left(\chi^{2}(4)=14,24, p=0,007\right)$. O ranqueamento ordenou de forma crescente os tipos de história de acordo com o tempo médio de pausas silentes, da seguinte forma: mecânica 1, comportamental 2, intencional, mecânica 2 e comportamental 1.

Foi possível verificar que o tipo de história não influencia o tempo médio de pausas silentes durante a produção de narrativas para as crianças com distúrbio específico de linguagem.

\section{Comparação entre o desempenho médio dos grupos}

A comparação entre os grupos indica diferença em todos os tipos de narrativas com maior média no grupo com DEL (Tabela 4).

Tabela 1. Análise descritiva da média de tempo das pausas pelo tipo de história no grupo com desenvolvimento típico de linguagem

\begin{tabular}{|c|c|c|c|c|c|c|c|}
\hline Tipo de história & Média & Mínimo & Máximo & Desvio-padrão & 25으 percentil & Mediana & 75으 percentil \\
\hline Mecânica 1 & 125,43 & 18,58 & 589,05 & 108,49 & 67,72 & 92,43 & 163,20 \\
\hline Mecânica 2 & 94,73 & 15,62 & 443,76 & 73,65 & 48,74 & 78,35 & 112,13 \\
\hline Comportamental 1 & 115,80 & 23,04 & 358,68 & 65,93 & 73,61 & 101,11 & 151,17 \\
\hline Comportamental 2 & 90,73 & 12,77 & 272,63 & 47,93 & 57,18 & 88,43 & 106,89 \\
\hline Intencional & 89,23 & 25,73 & 286,99 & 48,91 & 56,84 & 87,42 & 104,44 \\
\hline
\end{tabular}


Tabela 2. Comparação entre o tipo de história no grupo com desenvolvimento típico de linguagem

\begin{tabular}{lcccc}
\hline Comparações & $\mathrm{T}$ & $\mathrm{z}$ & $\mathrm{R}$ & Valor de p \\
\hline Mecânica 2 x Mecânica 1 & 165,00 & $-3,293$ & $-0,37$ & $0,001^{*}$ \\
Comportamental 1 x Mecânica 1 & 399,00 & $-0,148$ & $-0,02$ & 0,889 \\
Comportamental 2 x Mecânica 1 & 202,00 & $-2,796$ & $-0,31$ & $0,004^{*}$ \\
Intencional x Mecânica 1 & 206,00 & $-2,742$ & $-0,31$ & $0,005^{*}$ \\
Comportamental 1 x Mecânica 2 & 172,00 & $-3,199$ & $-0,36$ & $0,001^{*}$ \\
Comportamental 2 x Mecânica 2 & 377,00 & $-0,444$ & $-0,05$ & 0,666 \\
Intencional x Mecânica 2 & 366,00 & $-0,591$ & $-0,07$ & 0,563 \\
Comportamental 2 x & & & & \\
Comportamental 1 & 171,00 & $-3,212$ & $-0,36$ & $0,001^{*}$ \\
Intencional x Comportamental 1 & 136,00 & $-3,683$ & $-0,41$ & $<0,001^{*}$ \\
Intencional x Comportamental 2 & 379,00 & $-0,417$ & $-0,05$ & 0,685 \\
\hline
\end{tabular}

*Valores significativos $(p \leq 0,05)$ - Teste de postos com sinais de Wilcoxon

Legenda: $T$ = estatística para o teste de postos de com sinais de Wilcoxon; $z=u m$ ponto de dados expresso em unidades de desvio-padrão; $\mathrm{R}$ = tamanho do efeito
Tabela 4. Comparação da média de tempo das pausas pelo tipo de história entre os grupos

\begin{tabular}{lcccc}
\hline Tipo de história & Grupo & Média & z & Valor de p \\
\hline \multirow{2}{*}{ Mecânica 1 } & Controle & 25,88 & $-2,901$ & $0,004^{*}$ \\
& Pesquisa & 39,75 & & \\
Mecânica 2 & Controle & 23,25 & $-4,548$ & $<0,001^{*}$ \\
& Pesquisa & 45,00 & & \\
Comportamental 1 & Controle & 23,33 & $-4,501$ & $<0,001^{*}$ \\
& Pesquisa & 44,85 & & \\
Comportamental 2 & Controle & 23,38 & $-4,469$ & $<0,001^{*}$ \\
& Pesquisa & 44,75 & & \\
Intencional & Controle & 23,00 & $-4,704$ & $<0,001^{*}$ \\
& Pesquisa & 45,50 & &
\end{tabular}

*Valores significativos $(p \leq 0,05)$ - Teste de Mann-Whitney

Legenda: $z=$ um ponto de dados expresso em unidades de desvio-padrão

Tabela 3. Análise descritiva da média de tempo das pausas pelo tipo de história no grupo com distúrbio específico de linguagem

\begin{tabular}{lccccccc}
\hline Tipo de história & Média & Mínimo & Máximo & Desvio-padrão & 25o percentil & Mediana & $75^{\circ}$ percentil \\
\hline Mecânica 1 & 209,65 & 37,39 & 525,06 & 128,27 & 97,55 & 174,08 \\
Mecânica 2 & 197,54 & 55,94 & 474,50 & 101,01 & 116,39 & 174,02 & 244,48 \\
Comportamental 1 & 247,83 & 102,56 & 754,97 & 147,46 & 154,88 & 201,52 & 307,41 \\
Comportamental 2 & 199,91 & 62,52 & 503,94 & 103,27 & 119,04 & 198,88 & 239,08 \\
Intencional & 194,74 & 42,12 & 408,43 & 88,23 & 116,48 & 194,64 & 251,00 \\
\hline
\end{tabular}

\section{DISCUSSÃO}

Ao comparar o desempenho de crianças em desenvolvimento típico de linguagem com aquelas com DEL foi possível perceber que a média das pausas silentes foi maior para o segundo grupo.

Como as pausas silentes tendem a ocorrer quando o falante enfrenta alguma dificuldade durante a formação de conceitos ou na recuperação das estruturas linguísticas para a produção da fala ${ }^{(22)}$, este resultado nos permite afirmar que nos quadros de DEL a ocorrência de pausas mais longas confirma a fragilidade do processamento de linguagem ${ }^{(4,5)}$.

Este grupo precisa se esforçar mais do que as crianças em desenvolvimento típico de linguagem em tarefas que envolvem a produção linguística. Dessa forma, a duração das pausas silentes é mais longa nesta população, reforçando a presença de uma sobrecarga no sistema linguístico ${ }^{(4,23)}$.

A investigação do tempo médio da pausa silente indicou que em ambos os grupos a menor média ocorreu nas histórias de tipo intencional, achado que difere do que supúnhamos devido ao fato deste modelo de história envolver a atribuição de estados mentais.

A comparação destes grupos também nos permitiu perceber que o aumento da complexidade da história influencia a ocorrência de pausas silentes apenas nas narrativas de crianças sem alteração de linguagem. Uma explicação possível para este achado reside no fato da população com DEL ter prejuízos para descrever figuras, manter o tópico conversacional, sequenciar eventos e apresentar explicações, habilidades que estão intrinsecamente relacionadas às habilidades narrativas ${ }^{(24)}$.

Todavia é interessante notar que as crianças em desenvolvimento típico de linguagem tiveram pausas silentes mais curtas na elaboração das narrativas intencionais e das mecânicas 2 . $\mathrm{O}$ esperado era que as pausas fossem mais curtas nas narrativas mecânicas, pois são menos complexas, visto que envolvem apenas a interação casual de pessoas e/ou objetos; e mais longas nas narrativas intencionais, mais complexas por envolverem a atribuição de estados mentais a pessoas em atividades cotidianas ${ }^{(20)}$.

Tal achado pode ser explicado pelo fato dos sujeitos da pesquisa encontrarem-se na faixa etária entre sete e dez anos, período no qual importantes aquisições linguísticas determinam o refinamento das habilidades narrativas. Logo, apesar da narrativa intencional ser considerada uma tipologia complexa, é possível que a curta duração da pausa tenha sido influenciada por um lado pela simplificação da narrativa elaborada pelos sujeitos, mas por outro pelo fato deles estarem habituados a relatar fatos cotidianos que envolvem intencionalidade, o que amenizaria sua suposta complexidade ${ }^{(25,26)}$.

No caso das crianças com DEL, a ausência dessa influência sugere que elas tenham dificuldades mais abrangentes e não restritas apenas à complexidade, visto que a elaboração de narrativas equivale a uma tarefa complexa que integra simultaneamente diversas competências linguísticas. De fato, estas crianças apresentam prejuízo em aspectos pragmáticos relativos à identificação do tema principal de conversas e história ${ }^{(24)} \mathrm{e}$ em aspectos linguísticos relacionados principalmente à elaboração expressiva. Tais prejuízos comprometem de maneira significativa o discurso, uma vez que as dificuldades linguísticas se expressam desde em uma limitação no vocabulário até a dificuldade de compreensão e domínio das regras da língua ${ }^{(13,16)}$.

Em estudo anterior com a mesma população foi verificado que o grupo com DEL apresentou mais erros na ordenação 
temporal das figuras que embasam a história, porém tal diferença ocorreu apenas para as narrativas mecânicas e intencionais ${ }^{(27)}$. Como as pausas mais longas neste grupo ocorreram nas narrativas comportamentais não é possível estabelecer relações causais diretas, mas é necessário proceder a uma investigação mais detalhada para esclarecer se a ordenação das figuras pode interferir na fluência de fala deste grupo.

Estes achados sugerem, portanto, que a população em questão pode enfrentar dificuldades no estabelecimento de um diálogo, pois o prejuízo de suas habilidades conversacionais, aliado ao aumento de disfluências, tende a favorecer que o interlocutor perca o interesse na mensagem. Assim, é importante que o terapeuta considere também as habilidades sociais durante o processo de reabilitação de um quadro de alteração de linguagem e busque desenvolver estratégias que tornem esta criança um falante mais ativo.

É interessante apontar ainda que a fluência de fala se mostrou eficiente na distinção destes grupos, ainda que este não fosse o objetivo principal do estudo. Por fim, novas pesquisas que esclareçam a relação entre a complexidade da história, a ocorrência e duração das pausas silentes, e as características da narrativa elaborada pelos sujeitos de ambos os grupos podem ampliar a compreensão da ausência dessa influência nos indivíduos com DEL e nos trazer indicativos de como intervir nesta população.

\section{CONCLUSÃO}

As crianças com DEL, devido ao seu comprometimento linguístico, apresentaram pausas silentes mais longas em suas narrativas. A complexidade da história influenciou o tempo médio da pausa silente durante a produção das narrativas das crianças em desenvolvimento típico de linguagem, porém esta influência não ocorreu nas crianças com DEL.

*PRP e LBB foram responsáveis pela coleta e tabulação dos dados; AMCA supervisionou a coleta de dados e colaborou com a análise dos dados; DMBL foi responsável pelo projeto e delineamento do estudo e orientação geral das etapas de execução e elaboração do manuscrito.

\section{REFERÊNCIAS}

1. Price JR, Roberts JE, Jackson SC. Structural development of the fictional narratives of African American preschoolers. Lang Speech Hear Serv Sch. 2006;37(3):178-90.

2. Seiger-Gardner L, Schwartz RG. Lexical access in children with and without specific language impairment: a cross-modal picture-word interference study. Int J Lang Commun Disord. 2008;43(5):528-51.

3. Andrade CRF. Abordagem neurolinguística e motora da gagueira. In: Ferreira LP, Befi-Lopes D, Limongi SCO (Org.). Tratado de Fonoaudiologia. São Paulo: Roca; 2004. p. 1001-16.

4. Guo LY, Tomblin JB, Samelson V. Speech disruptions in the narratives of English-speaking children with specific language impairment. J Speech Lang Hear Res. 2008;51(3):722-38.

5. Hall NE. Lexical development and retrieval in treating children who stutter. Lang Speech Hear Serv Sch. 2004;35(1):57-69.
6. Sormani I. Development of speech disruptions in narratives of dutch-speaking children with specific language impairment: Universiteit Utrecht; 2010.

7. Rispoli M, Hadley P. The leading-edge: the significance of sentence disruptions in the development of grammar. J Speech Lang Hear Res. 2001;44(5):1131-43.

8. Rispoli M. Changes in the nature of sentence production during the period of grammatical development. J Speech Lang Hear Res. 2003;46(4):818-30.

9. Gray S. Word-learning by preschoolers with specific language impairment: what predicts success? J Speech Lang Hear Res. 2003;46(1):56-67.

10. Gershkoff-Stowe L, Hahn ER. Fast mapping skills in the developing lexicon. J Speech Lang Hear Res. 2007;50(3):682-97.

11. Capone NC, McGregor KK. The effect of semantic representation on toddlers' word retrieval. J Speech Lang Hear Res. 2005;48(6):1468-80.

12. Bishop DV. The underlying nature of specific language impairment. J Child Psychol Psychiatry. 1992;33(1):3-66.

13. Befi-Lopes DM. Avaliação diagnóstica e aspectos terapêuticos nos distúrbios específicos de linguagem. In: Fernandes FD, Mendes BC, Navas AL (eds.). Tratado de Fonoaudiologia. $2^{\circ}$ ed. São Paulo: Roca; 2010. p. 314-22.

14. Ukrainetz TA, Gillam RB. The expressive elaboration of imaginative narratives by children with specific language impairment. J Speech Lang Hear Res. 2009;52(4):883-98.

15. Araujo K. Desempenho gramatical de criança em desenvolvimento normal e com distúrbio específico de linguagem. São Paulo: Universidade de São Paulo; 2007.

16. Befi-Lopes DM, Bento AC, Perissinoto J. Narration of stories by children with specific language impairment. Pro Fono. 2008;20(2):93-8.

17. Wertzner HF. Fonologia. In: Andrade CRF, Befi-Lopes DM, Fernandes FDM, Wertzner HF (eds.). ABFW: teste de linguagem infantil nas áreas de fonologia, vocabulário, fluência e pragmática. $2^{\mathrm{a}}$ ed. Barueri: PróFono; 2004. p. 5-32.

18. Andrade C, Befi-Lopes D, Fernandes F, Wertzner H. Manual de avaliação de linguagem do serviço de fonoaudiologia do Centro de Saúde Escola Samuel B. Pessoa. São Paulo: Centro de Saúde Escola Samuel B. Pessoa; 1997. p. 127.

19. Andrade CRF, Befi-Lopes DM, Fernandes FDM, Wertzner HF (eds.). ABFW: teste de linguagem infantil nas áreas de fonologia, vocabulário, fluência e pragmática. $2^{\mathrm{a}}$ ed. Barueri: Pró-Fono; 2004.

20. Baron-Cohen S, Leslie A, Frith U. Mechanical, behavioural and intencional understanding of stories in autistic children. Br J Dev Psychol. 1986;4:113-25.

21. Perissinoto J. Avaliação fonoaudiologica da criança com autismo. In: Perissinoto $\mathrm{J}$ (ed.). Conhecimentos essenciais para atender bem a criança com autismo. São José dos Campos: Editora Pulso; 2003. p. 45-55.

22. Postma A, Kolk H. The covert repair hypothesis: prearticulatory repair processes in normal and stuttered disfluencies. J Speech Hear Res. 1993;36(3):472-87.

23. Boscolo B, Ratner N, Rescorla L. Fluency of school-aged children with a history of specific expressive language impairment: an exploratory study. Am J Speech Lang Pathol. 2002;11(1):41-9

24. Osman DM, Shohdi S, Aziz AA. Pragmatic difficulties in children with specific language impairment. Int J Pediatr Otorhinolaryngol. 2011;75(2):171-6.

25. Befi-Lopes DM, Paula EM de. Habilidades de resolução de conflito e ocorrência de disfluências comuns em crianças em desenvolvimento normal de linguagem. Rev Soc Bras Fonoaudiol. 2008;13(3):272-8.

26. Paula EM de, Befi-Lopes DM. Estratégias de resolução de conflito em crianças em desenvolvimento normal de linguagem: cooperação ou individualismo? Rev Soc Bras Fonoaudiol. 2011;16(2):198-203.

27. Bento ACP. Organização e narração de histórias por escolares em desenvolvimento típico de linguagem e com distúrbio específico de linguagem (DEL). Rev Soc Bras Fonoaudiol. 2010;15(4):628. 